\title{
SALUTIS ENIM PUBLICAE MAXIMA SEMPER RATIO HABENDA EST: LEIBNIZ Y LA TRADICIÓN SALMANTINA SOBRE LA MORALIDAD DE LOS SEGUROS
}

\section{SALUTIS ENIM PUBLICAE MAXIMA SEMPER RATIO HABENDA EST: LEIBNIZ AND THE SCHOOL OF SALAMANCA ON THE MORALITY OF INSURANCES}

LEONARDO RUIZ-GÓMEZ

Universidad Panamericana

Recibido: 03/07/2019_Aceptado: 23/09/2019

\section{RESUMEN}

Leibniz propone un sistema de seguros contra desastres naturales administrado por el Estado. Este sistema público de aseguramiento responde a una tradición de filosofía política, económica y ética que hunde sus raíces en la tradición escolástica y, de manera particular, en algunos representantes de la Escuela de Salamanca. El presente artículo pretende ofrecer un panorama general de la discusión tardomedieval en torno a los seguros y señalar dos problemáticas que resultan de particular relevancia: la relacionada con la usura y la relacionada con la providencia divina. A partir de este análisis, se explicarán las particularidades de la propuesta leibniziana, mostrando a la vez su originalidad y su vinculación con la tradición salmantina.

Palabras clave: Leibniz, moral económica, providencia, seguros, usura. 


\section{ABSTRACT}

Leibniz proposed a natural disaster insurance coordinated by the government. This proposal was aligned with a tradition of political, economic and ethical thought that is grounded in scholastic philosophy and, particularly, in the School of Salamanca. This paper aims to offer a general view of the discussion in late-medieval philosophy regarding insurance; the analysis will point out two troublesome issues: the problem of usury and the problem of divine providence. From this, I will explain some particularities of the Leibnizian proposal on the scope of the late medieval debate by showing its originality and relation with the School of Salamanca.

Keywords: business ethics, insurances, Leibniz, providence, usury.

\section{INTRODUCCIÓN}

Gottfried Wilhelm Leibniz (1646-1716) pertenece indudablemente a los anales de tan diversas disciplinas y ciencias que es difícil encontrar rincones en la historia del pensamiento en donde no se vea su huella. Aunque es sumamente reconocido por sus trabajos en matemáticas, ciencia natural y metafísica, no podemos olvidar que Leibniz era, antes que nada, un doctor en derecho, un hombre interesado tanto en la esencia misma de lo justo y de la ley, como en los asuntos del ámbito público y político. Todo proyecto del filósofo alemán, ya fuera teórico o práctico, estaba encaminado a un solo propósito: concretizar las condiciones de posibilidad que, eventualmente y con la colaboración de toda la humanidad, podrían llevar al género humano y a sus sociedades a la paz y el bien perpetuos.

Si bien esta desiderata puede sonar un tanto utópica en nuestros tiempos, es importante reconocer que Leibniz fue ideando dispositivos concretos y prácticos que abonarían a este fin. Uno de estos mecanismos políticos que Leibniz veía en conexión con el bien común es el de las instituciones aseguradoras. Estas figuras financieras tenían ya una larga historia antes de que el filósofo alemán se ocupara de ellas; sin embargo, sus trabajos en torno a ellas resultaron en aportaciones considerables tanto en el ámbito matemático como en el político.

En el presente trabajo expondré un breve panorama histórico de las complicaciones morales, políticas y legales que la tradición escolástica y, principalmente, la escuela salmantina heredó a la cosmovisión protestante del siglo XVII. A partir de este análisis, mostraré cómo la concepción política que tiene Leibniz de las instituciones aseguradoras responde a esta tradición que, como se verá, 
genera una doble problemática sobre la que Leibniz tendrá que trabajar. A pesar de que no hace Leibniz un estricto análisis moral del asunto (o precisamente porque no lo hace), el objetivo de este artículo es relacionar algunas de las tesis políticas y éticas del autor para dar una visión integral y coherente del papel que juegan las aseguradoras dentro de su plan político. Se mostrará que, a partir del tratamiento de algunos autores salmantinos y, principalmente, Domingo de Soto, surgen dos complicaciones morales inherentes a la cuestión de los seguros. Por una parte, el tema de la providencia; por otra, el de la usura. Ambas complicaciones tendrán ecos en la tradición tardoescolástica católica y protestante. Leibniz tendrá que hacerse cargo de ambas a través de la noción de bien común y de su particular idea de providencia divina. Si bien nuestro autor es bien conocido por sus aportes matemáticos en materia financiera, vale la pena aclarar que no se abordarán en el presente trabajo los modelos estadísticos desarrollados por Leibniz. $^{1}$

Una última aclaración merece la pena ser mencionada: no es el objetivo de este trabajo demostrar conexiones directas entre autores particulares, sino mostrar la manera en la que la tradición salmantina, una escuela que Leibniz conocía bien, generó ciertos conceptos y problemáticas que influyeron, de manera más o menos directa, en el pensamiento del autor alemán. Antes de entrar de lleno al tema de la moralidad de los seguros, me permitiré hacer una breve anotación sobre la relación que tiene Leibniz con la tradición escolástica. Ésta servirá como contexto hermenéutico para justificar los puentes y nexos entre la tradición tardomedieval y el pensamiento leibniziano.

\section{LEIBNIZ Y LA ESCOLÁSTICA}

A pesar de la innegable influencia que tuvo la filosofía renacentista y tardomedieval en la así llamada "filosofía de la modernidad temprana", no es fácil seguir la pista de estas conexiones a través de los textos modernos. Personajes como Descartes, Spinoza y Locke no revelan de manera explícita sus fuentes y, al contrario, suelen presentar sus ideas como si su pensamiento no tuviera antecedentes en la tradición filosófica; las pocas referencias a la escolástica suelen ser condescendientes o francamente negativas. Un caso muy distinto es el de Leibniz, probablemente el filósofo que reconoce más explícitamente su deuda con las tradiciones filosóficas que le preceden. Sin embargo, no se trata meramente de un asunto de reconocimiento: difícilmente se podría encontrar un autor

1 Análisis de las matemáticas que aporta Leibniz en el contexto de los contratos de seguros se pueden encontrar en Knobloch 2017, 2016; De Mora 2002. 
moderno con un conocimiento tan vasto de tantas obras, autores y corrientes de pensamiento como el del autor alemán.

En este sentido, la tradición escolástica y, en concreto, los grandes autores de la Escuela de Salamanca no pasaron en absoluto desapercibidos. Ya desde textos tan tempranos como la Disputatio metaphysica de principio individui, publicado apenas un mes después del cumpleaños diecisiete de Leibniz, ${ }^{2}$ constatamos un conocimiento profundo de la obra de Aquino, Escoto, Suárez, Ockham y un sinfín de otros autores escolásticos. ${ }^{3}$

En algunos textos, su evaluación general de la tradición escolástica no era muy distinta a la de sus contemporáneos, ya que solía expresarse negativamente de ella como un saber estéril. Basta observar el siguiente fragmento de 1671 en el que, con un tono evidente de petulancia, afirma:

Cuando imbuido de un espíritu semejante [habla de sí mismo], asistía según la costumbre a la reunión de sus pares, se le consideraba un monstruo. En efecto, él, que había sabido penetrar sin ayuda en los secretos de la filosofía y la teología escolástica, que entonces pasaba vulgarmente por ser el ápice de la sabiduría, presentaba en forma fácil y accesible su contenido, el cual constaba únicamente de conceptos. Por lo demás, sentía desdén por la filosofía y la teología escolástica y las juzgaba superficiarias e inútiles para el progreso humano (A VII, 2, 511 / Olaso, 49).

Esta valoración tan negativa se ve frecuentemente matizada cuando habla de algún autor particular y, sobre todo, cuando reconoce, en etapas más tardías de su vida, que su filosofía dio un giro fundamental cuando redescubrió, en el seno de su metafísica, las formas sustanciales de la escolástica aristotélica.

2 Un texto de 1676 deja ver que, desde sus 12 años de edad, Leibniz ya estaba familiarizado con la obra de los grandes autores de las escuelas salmantina y conimbricense: "Entretanto, me ocupaba de Zabarella, de Rubio, de Fonseca y de otros escolásticos, experimentando tanto placer como antes con los relatos históricos y llegué al punto de leer a Suárez con la misma facilidad con que solemos leer las fábulas milesias, que vulgarmente suelen ser llamadas novelas" (K I, 36-7 / Olaso, 61). Para las obras de Leibniz se emplearán las siguientes ediciones y abreviaturas: AA (Leibniz 1923), GP (Leibniz 1965), Knob. (Leibniz 2000), K (Leibniz 1864), Grua (Leibniz 1948), OFC (Leibniz 2007), Guillén (Leibniz 1991), Olaso (Leibniz 1982), Andreu (Leibniz 2001). Si no se señala nada, las traducciones son mías.

3 Se trata de un texto de apenas siete folios en el que se refiere, además de los mencionados, a autores tan dispares como Pedro Aureolo, Hervé de Nédellec, Paolo Soncinas, Gregorio de Rímini, Gabriel Biel, Fulgencio Schautheet, Durando de Saint-Pourçain, Murcio de la Llana, Christopher de Ramoneda, Marco Antonio Zimara, Abraham Calov, Daniel Stahl, Juan de Bassolius, Pedro de Fonseca, Dionysius von Rickel, Perdo de Posnania, Giacomo Zabarella, Agustín Nifo Suésano, Antonio Rubio, etc. Si bien en esta lista se incluyen autores de diversas tradiciones, el texto podría pasar como un intento tardoescolástico de solucionar el famoso problema de la individuación. Se trataba, por cierto, de su tesis de licenciatura, dirigida por su profesor, mentor y amigo Jakob Thomasius. 
Leibniz relata, en una famosa carta a Nicolás Remond de 1714 (GP III, 605-7), que siendo un infante "aprendí a Aristóteles, e incluso los escolásticos no me disgustaban". Tiempo después -continúa en la misma carta-, mientras paseaba por el bosque de Rosental en Leipzig, decidió, con apenas quince años, abandonar las escuelas y abocarse al estudio de la filosofía mecanicista y la matemática. Será hasta el fin de su estancia en París que "regresará a las entelequias" cuando se dé cuenta de que la matemática es insuficiente para explicar las razones últimas del mecanicismo. Estas formas sustanciales o entelequias suelen estar referidas a la filosofía aristotélica; sin embargo, es evidente que Leibniz tiene a la tradición escolástica en mente. ${ }^{4}$ En el ámbito teológico y moral, la cercanía con estas tradiciones es todavía más evidente. Basta revisar la Teodicea $a^{5}$ para constatar su vastísimo conocimiento de autores medievales y tardoescolásticos; en efecto, la cantidad de referencias que hace a estos autores a lo largo de toda su obra es notable. ${ }^{6}$

En general, hay un consenso creciente entre los comentadores de tomar con cautela las propias palabras de Leibniz. Se suelen matizar las etapas que él mismo, al final de su vida, relata en la carta a Remond y en algunos otros documentos. Es cada vez más claro, por la evidencia textual surgida, que Leibniz nunca abandonó del todo el aristotelismo y el escolasticismo de sus etapas más tempranas, y que el "resurgimiento" de estas ideas aristotélicas no es sino una reformulación de ideas, conceptos y tesis que permanecieron siempre activas en la mente del filósofo de Leipzig (Garber 2011, 7; Mercer 1997). ${ }^{7}$ Esto es relevante para el análisis que sigue, pues algunas de las reflexiones en torno a los seguros, las rentas vitalicias o los montes de piedad corresponden precisamente a esta etapa en la que Leibniz se encuentra supuestamente "alejado" de la filosofía de las escuelas y sumido en elaboraciones matemáticas indispensables para

4 Cfr. por ejemplo, Système nouveaux pour expliquer..., GP IV, 474; Système nouveaux de la nature..., GP IV, 479. No hay que olvidar que la lectura leibniziana de Aristóteles y de los escolásticos está fuertemente influenciada por la visión de su maestro Thomasius, quien era de la opinión de que entre el estagirita y los escolásticos mediaba una distancia semejante a aquella entre el gran hombre de estado y el monje encerrado en su celda (Cfr. Pelletier 2014, 123).

5 En esta misma obra se observa la ambivalente opinión de Leibniz: mientras cita un ingente número de autores escolásticos, se atreve a soltar la siguiente sentencia: "A veces hay oro oculto bajo las basuras del latín bárbaro de los monjes” (Theodicée, GP VI, 53 / OFC 39).

6 A lo largo del texto presentaré evidencia textual, en caso de haberla, del conocimiento directo que tenía Leibniz de cada uno de los autores mencionados. Esto no implica, como mencioné en la introducción, que afirme que hay una relación directa en el tema de las instituciones aseguradoras, sino que muy probablemente Leibniz estaba al tanto de las ideas que estos autores habían desarrollado en torno a este tema.

7 Para profundizar en la relación entre Leibniz y los escolásticos, se puede confrontar el volumen 46(2) de Studia Leibnitiana, editado por Arnaud Pelletier y dedicado en su totalidad a este tema. 
los cálculos financieros involucrados en estos asuntos. Como se verá a continuación, Leibniz no es de ninguna manera ajeno a las reflexiones escolásticas sobre los asuntos morales que rodean a las instituciones aseguradoras.

\section{CONTEXTO HISTÓRICO: LOS SEGUROS EN EL SIGLO XVII}

Los contratos de seguro aparecieron probablemente en Italia a finales del siglo XIII o comienzos del siglo XIV. La póliza más antigua de la que se tiene noticia data de 1343 (Ceccarelli 2001, n. 63). Sin embargo, el uso cotidiano de este tipo de contratos iba acompañado por un incesante debate sobre su legitimidad y moralidad. Tanto la discusión filosófica como la práctica habitual de los contratos de seguro llegaron hasta al siglo XVII, siglo en el que empezaron a proliferar las compañías de este tipo.

En la literatura filosófica del medioevo tardío, el ejemplo paradigmático en la discusión sobre los contratos de seguros es el del seguro mercantil, principalmente, el relacionado con el comercio marítimo. Se solían celebrar contratos entre particulares en los que el asegurante, a cambio de una cantidad relativamente baja, se hacía cargo del riesgo que implicaba llevar una nave a alta mar. En ocasiones se aseguraba la mercancía, en ocasiones la nave, e incluso a veces se aseguraban financiamientos a los transportistas (préstamos a la gruesa) (Cruz Barney 1998, 111-12).

A mediados del siglo XVII, aparecen en Inglaterra contratos privados de aseguramiento, ya no con fines mercantiles. El ejemplo paradigmático es el contrato de protección contra incendios y, en menor grado, el de desastres relacionados con el agua. La guerra de los treinta años y, principalmente, el gran incendio de 1666 en Londres (en el que cerca de 13,000 casas fueron consumidas por el fuego) evidenciaron la necesidad de generar un esquema en el que el patrimonio de los ciudadanos no quedara a merced de las vicisitudes del clima y de la negligencia humana. Tan sólo un año después del gran incendio, se abrió la primera compañía de seguros privada en Inglaterra y pronto empezaron a proliferar compañías conforme se aproximaba el fin de siglo (Thomann y von der Schulenburg 2010, 46). Esta dinámica se extenderá a lo largo del siglo XVIII, en el que los incendios continuarán azotando las ciudades inglesas a pesar de los esfuerzos gubernamentales por modificar los criterios de construcción y el comportamiento descuidado de los ciudadanos. ${ }^{8}$

8 Como ejemplo de este esfuerzo gubernamental se encuentra la Rebuilding Act de 1667, que modificaba los criterios de construcción para los edificios de Londres, beneficiando la piedra sobre la 
Leibniz visitará por primera vez Londres en el año 1673, sólo siete años después del devastador incendio. Los estragos de éste eran todavía evidentes en la capital inglesa y no hay duda alguna de que se habría informado de los esquemas de aseguramiento que estaban apareciendo en Londres.

Después de su estancia en París, Leibniz pasa a formar parte de la corte de Hannover a finales de 1676. Ese mismo año, un devastador incendio había arrasado con el poblado de Oldenburg, perteneciente al electorado de Hannover. Las pertenencias no estaban aseguradas, pues el conde Anton Günther de Oldenburg-Delmenhorst había rechazado, por cuestiones morales y religiosas, el ofrecimiento de parte de Wilhelm Stiell, comerciante de Hamburgo, de asegurar parte de la ciudad. El conde argumentaba que un seguro contra incendios implicaba un acto contra la providencia divina; una opinión que, como se verá, era habitual en la época (Schulenburg; Matthias, 47). ${ }^{9}$

Estos acontecimientos llevaron con seguridad a Leibniz a considerar el aseguramiento de las ciudades como una vía para garantizar bienestar a los ciudadanos. Sin embargo, Leibniz no va a considerar exclusivamente las ventajas prácticas de un modelo de aseguramiento de inmuebles sin tomar en cuenta sus complicaciones morales, jurídicas y políticas. Pero sobre estos temas hay una larga discusión histórica de la que Leibniz estaba advertido y a la que tendría que dar respuesta.

\section{LOS SEGUROS EN LA ESCUELA DE SALAMANCA Y EL MUNDO PROTESTANTE}

Ceccarelli señala a Bartolomeo de San Concordio como el primero en hacer, en torno a 1338, una reflexión directa sobre los contratos de seguros en la Edad Media (Ceccarelli 2001, n. 63). Desde ahí, la discusión moral sobre éstos no encontrará consenso sino hasta bien entrado el siglo XVIII. De hecho, en el siglo XVII las aseguradoras seguían recibiendo acusaciones de inmoralidad en Inglaterra y, como ya hemos visto, lo mismo pasaba en el Imperio Romano Germánico (Rossi 2016, 10).

madera (Thomann and von der Schulenburg 2010, 46). Sin embargo, como demuestra Robin Pearson, los incendios continuaron propagándose a largo del siglo XVIII a una ratio de aproximadamente 153 casas destruidas por año en Gran Bretaña (Pearson 2010, 76). Esto explica por qué la celebración de contratos de seguros no esperó a un consenso en la discusión filosófica y moral que había principalmente entre los teólogos protestantes.

9 En medio de este debate teológico, en 1676 se fusionaron en Hamburgo cuarenta y seis contratos de aseguramiento contra incendios en el Fondo General para Incendios. Otro suceso del que sin duda Leibniz tuvo noticia (Schulenburg; Matthias, 47). 
La discusión moral sobre los seguros en el siglo XVII se enmarcaba en dos frentes distintos. Por una parte, se encontraba la acusación de usura, puesto que el asegurador parecía beneficiarse económicamente sin haber prestado un servicio real, sino la mera posibilidad de disponer de su capital en el futuro. En segundo término, la discusión estaba vinculada a la providencia divina, pues el asegurador lucraba con eventos futuros, cuya verificación parecía azarosa y sólo accesible a la mente divina. Sin embargo, como ya se dijo, la realidad de este tipo de contratos permaneció de manera continua a lo largo de los años a pesar de los reparos teológicos y morales. Los juristas en ocasiones lo equiparaban a una simple apuesta, cuando se acusaba de usura a las aseguradoras; en cambio, cuando en la sociedad se censuraba fuertemente el juego de azar, los juristas acercaban el contrato de seguros a un simple contrato entre particulares, disminuyendo el protagonismo del azar en la transacción (Rossi, 10).

En este sentido, el estatuto moral de los seguros estaba a merced de las reflexiones de los filósofos y teólogos en torno a la usura y los juegos de azar. Ambos temas fueron prolijamente trabajados en la Escuela de Salamanca y en las tradiciones posteriores y tuvieron una relevancia central en las discusiones económicas del siglo XVII.

\section{LA USURA}

En el año 1234, el papa Gregorio IX publicó la decretal pontificia Naviganti, donde se juzgaba como usura el hecho de que un prestamista aportara un capital que, por el riesgo que éste correría, recibiera intereses más altos. ${ }^{10}$ Esto es, se censuraba la idea de que el prestamista absorbiera el riesgo a cambio de recibir un beneficio económico sobre éste. Así, las aseguradoras, que intentaban también lucrar con el riesgo, caerían prima facie bajo esta acusación de usura.

El problema de la usura tiene una larga tradición dentro de los autores católicos del medioevo. Además de las prohibiciones explícitas en el Antiguo Testamento, los filósofos católicos argumentaban que el cobro de intereses implicaba cobrar el dinero entregado y, además, el uso del mismo (de ahí el término usura). Desde Aristóteles (1257a, 35), se consideraba al dinero como estéril y, en consecuencia, su uso (gasto o inversión) no podía separarse de su naturaleza;

10 "Usurero es el que recibe de un deudor algo más del capital, aunque asuma él mismo (en su persona) el riesgo. H. d primero. No es usurero el que compra una cosa en menos del justo precio si en ese momento se duda realmente si la cosa valdrá más en el momento del pago o menos. $H$. $d$. segundo. En caso de duda queda excusado el que por diferir el pago del precio vale la cosa en más de lo que vale si no estaba por venderse H. d. tercero" (Gregorio IX, l. V, tit. XIX, De usuris). 
entonces, cobrar por el uso del dinero era cobrar dos veces por la misma cosa, o bien, cobrar por algo inexistente (Cfr. Aquino, II-II, q. 78, a. 1).

La pregunta es, entonces, en qué lugar entra aquí el contrato de seguros. Los seguros marítimos consistían en un contrato de compra-venta: el asegurador era el comprador, el asegurado el vendedor; así, la transacción se efectuaba si, y sólo si, la mercancía resultaba dañada, si era entregada a piratas o si tenía que lanzarse por la borda para salvar la embarcación. Si la mercancía resultaba inerme durante el viaje, el asegurado recuperaba la propiedad (Cruz Barney 1998, 171). Sin embargo, es fácil ver que un acuerdo de este tipo no sería posible si el asegurador no recibiera algo a cambio, incluso cuando dicha compra-venta no se efectuara. Se debe estipular entonces un objeto por el cual el asegurado paga ese dinero si no se quiere incurrir en usura.

Quien aborda con mayor claridad este asunto es Domingo de Soto ${ }^{11}$ en su célebre obra De iustitia et iure. Ahí, los seguros se ponen hombro con hombro con otro tipo de contratos como las loterías, las apuestas y las divisoriae sortes, contratos todos en donde el azar juega un papel preponderante (Soto, IV, q. 5, a. 2). Sin embargo, en la cuestión dedicada exclusivamente a los seguros, no aporta objeciones relacionadas con el carácter azaroso del asunto (Soto, VI, q. 7). Soto da tres contrargumentos: a) uno de autoridad, cuando se refiere al documento Naviganti antes mencionado; b) uno relacionado con la usura, pues quien recibe sobre sí un peligro a cambio de un precio "ni compra nada, ni entrega nada a la sociedad"; $y$, finalmente, c) un argumento consecuencialista, pues se permitiría que un hombre sin capital asegurara un objeto, ya que no se requiere el capital al momento del contrato, lo que podría generar un peligro para la sociedad. La respuesta de Soto a estas objeciones es contundente:

A esta cuestión se responde fácilmente con una conclusión afirmativa. El contrato de seguro es lícito, si se hace según ley y costumbre. La conclusión se demuestra por su objeto. Uno puede percibir remuneración por una cosa cualquiera que sea valorable; pues bien, poner en seguro una cosa, que se halla expuesta a peligro, tiene su valor; por consiguiente, este valor puede redimirlo por una cantidad, y por tanto percibir esta cantidad el que tome a su cargo tal peligro. Y ello principalmente porque ambos se someten al peligro, tanto el dueño de la nave al de pagar la cantidad, si la nave se salva, como el otro al de pagar las mercancías, si se pierden (Soto, VI, q. 7).

11 Leibniz cita directamente a Domingo de Soto en diversos lugares, principalmente en conexión con el tema de la gracia y la salvación. Cfr. De salvatione Gentium, AA IV, 5, 464, 466; Quaestio illustris, AA IV, 1, 452, 454; Aus und zu Isaac Papin, Essai de Theologie, AA VI, 2663. 
El contrato de seguros está libre de usura en la medida en que se ofrece un valor ("poner en seguro una cosa") por el cual se recibe dinero. Llama la atención la laxitud que muestra Soto en este asunto, y que no se observa en muchos otros temas vinculados a la usura. Basta revisar el capítulo sobre los Montes de Piedad, en donde Soto censura fuertemente que el prestamista cobre una cuota administrativa por el personal que resguardaría las prendas ofrecidas. Ni siquiera cuando se toma en consideración el bien que hace el Monte de Piedad a los más pobres relaja la censura, pues "por esta razón tendrías que defender también muchas otras usuras. Porque efectivamente, todos los que, obligados por la necesidad, reciben un préstamo con usura, reciben un beneficio gratuito" (Soto, VI, q. 1, a. 4). Sin embargo, en el caso de los seguros, Soto parece ser consciente de la necesidad que implica este seguro a la sociedad y se permite, en este caso sí, dar un argumento consecuencialista:

En segundo lugar, se arguye por la necesidad. Este contrato es muy necesario a la sociedad; y así, como por el objeto no adolece de vicio alguno, es bueno. $\mathrm{El}$ antecedente se demuestra claramente, porque es una ayuda necesaria para los negocios, ya que muchos mercaderes temerían cargar de mercancías sus naves con tanto peligro; más cuando por un precio reducido aseguran gran cantidad de riqueza, se deciden exponer a peligro tal mercancía, que es necesaria para la vida de la sociedad [quae quidem reipublicae necessaria est] (Soto, VI, q. 7).

Hay un cierto pragmatismo en el tono de Soto, quien parece reconocer la necesidad ineludible del comercio y el incentivo que supone que el comerciante no cargue con la totalidad del riesgo al que está sometido el comercio marítimo. Por esta razón, no titubea al apelar, en el indicio de la respuesta, al receptus usus, señalando la facticidad de este tipo de contratos.

Soto sintetiza una larga tradición de autores que admitían a los contratos de seguros como libres de usura (Bernardino de Siena, Lorenzo Ridolfi). El principal argumento consistía en desvincular a los seguros de la idea de préstamo y acercarlo más a la idea de un servicio. De esta manera, se salvaba a los seguros de la censura recibida por el decreto Naviganti (Ceccarelli 2001, 620-21). El caso más paradigmático sea quizá el de Martín de Azpilcueta, el doctor Navarrus, quien hace una larga disertación sobre cómo debe entenderse la decretal pontificia y las distintas lecturas (la de Soto incluida) que le antecedieron. ${ }^{12} \mathrm{Az}-$ pilcueta argumenta profusamente en su Comentario sobre la resolución del dinero que el decreto Naviganti sólo aplicaba a préstamos que demandaban un

12 Leibniz conocía la obra económica de Azpilcueta, como se puede ver en lo referente a las usuras en los procesos cambiarios, cfr. De subhastationibus, AA IV, 3, 756; Quaestio illustris, AA IV, 1,462 . 
retorno extra por asumir los riesgos de una empresa marítima, pero que no hacía referencia a un contrato de seguros. ${ }^{13}$

Sin embargo, la validación moral de los contratos de seguros no fue universal ni antes ni después de Domingo de Soto. Probablemente uno de los autores más representativos de la posición contraria es el teólogo alemán Conrad Summenhart. ${ }^{14}$ En su obra De contractibus licitis atque ilicitus, Summenhart critica duramente los contratos de seguros por no tener ninguna utilidad para la sociedad (respublica):

Además, tal contrato no importa utilidad alguna por sí misma a la sociedad; y si los contratantes realizan dicho contrato con la esperanza de lucrar, a manera de predicción, entonces no es sino querer nutrir a la sociedad sin ningún trabajo útil: pues en efecto cualquier hombre que necesita de bienes temporales debe adquirirlos a través de alguna acción útil a la sociedad, ya sea temporal o espiritual, lo cual sostengo a través de la autoridad y de la razón. Por la autoridad, en efecto, pues en Génesis III dice: "Con el sudor de tu frente ganarás tu pan”. Esta sentencia transita, no sobre la persona de Adán, sino sobre toda la naturaleza humana en él contenida [...]. La razón también enseña esto. Pues en el cuerpo natural no se le adjudica un acto a un miembro cualquiera, sino a aquél que reporta la utilidad para los otros miembros o para el cuerpo. En consecuencia, así también debe ser en el cuerpo místico de la sociedad, pues los actos de los hombres deben reportar utilidad a ésta (III, q. 71).

El problema con el contrato de seguro es que el asegurador no está generando ningún bien específico y en consecuencia se estaría cayendo en usura. ${ }^{15}$ Como señala Ceccarelli, son las mismas razones las que adjudican Soto y Summenhart para llegar a posiciones contrarias: es precisamente la utilidad a la respublica lo que avala o desacredita, en cada caso, el contrato de seguros (Ceccarelli 2001, 623).

Summenhart tendrá otras objeciones importantes contra las aseguradoras, pero éstas no están conectadas ya con el tema de la usura, sino con el de la providencia, que es el tema que se abordará a continuación.

13 Este texto es un apéndice de su célebre Manual de confesores y penitentes. Se puede encontrar una edición de este texto en Grabill (2007).

14 Leibniz cita directamente a Summenhart en Guilielmi pacidi. Plus ultra, AA VI, 4, 682.

15 Summenhart considera el caso en el que el asegurador se encarga personalmente de la seguridad del barco. Sólo en ese caso sería válido el contrato de seguro. 


\section{LA PROVIDENCIA}

Se dijo anteriormente que, para alejar al contrato de seguro de la noción de préstamo con usura, algunos autores, como Soto, equiparaban este tipo de contrato con las apuestas o los juegos de azar. Esto, sin embargo, no estaba en absoluto libre de complicaciones. De hecho, lo mismo que la usura, la apuesta era considerada un pecado. Sin embargo, esta vez, a diferencia de la usura, la apuesta tenía como particularidad que, aun considerada inmoral, admitía la discusión sobre si la adquisición de bienes por medio del azar estaba sujeta a retribución obligatoria. Había una tradición que sostenía que las apuestas (al igual que la prostitución, por cierto), aun siendo inmorales, no implicaban la liberación de la deuda (Aquino, II-II, q. 32, a. 7, ad. 2). La usura, en cambio, implicaba con necesidad la retribución de lo adquirido por estos medios, y la casuística moral se ocupaba de los detalles de los términos en que debía hacerse esta devolución (por ejemplo, si habían de devolverse o no los réditos que hubiera dado el dinero obtenido de esta manera).

Se encuentra desde el siglo XIII una vena franciscana que acerca los juegos de azar a cualquier tipo de contrato y busca, en este sentido, establecer un criterio de justicia en las partes que cada uno ha de pagar. Esto tiene como consecuencia, no sólo que se admita la legalidad de la explotación del riesgo, sino que se establezca un criterio de proporcionalidad entre la magnitud de dicho riesgo y la ganancia que generaría. El autor más destacado de esta tradición es Pedro Juan Olivi, contemporáneo del Aquinate, de quien se desprenderá una tradición que entiende los juegos de azar como un tipo más de contrato (Ceccarelli 2006). Esta tradición llegará hasta Domingo de Soto, cuya influencia, como ya se mencionó, será radical en la segunda mitad del siglo XVI y durante el siglo XVII. Soto condena el juego por distintas razones consecuencialistas (el vicio, el escándalo, el peligro), pero no pide que la transacción se restituya: "las cosas adquiridas con el juego pasan al dominio de quien las gana" (Soto, IV, q. 5, a. 2). Las razones son la libre voluntad del intercambio y la distribución homogénea del riesgo. Soto incluye aquí a los seguros como un caso análogo al de los juegos de azar y descarta, sin argumentar, el problema de la providencia divina en el asunto.

Sin embargo, la lectura contractualista de Olivi y de Soto sobre los juegos de azar se encontraba lejos de ser hegemónica. Ya desde el siglo XIV se mostraban objeciones importantes, principalmente de Bernardino de Siena y, posteriormente, de Conrad Summenhart (III, q. 71, 320-322). Esta tradición censuraba fuertemente los juegos de azar por su carácter aleatorio. En efecto, los eventos sobre los que se lucraba se encontraban sólo determinados en la mente divina 
$\mathrm{y}$, en consecuencia, cualquier contrato que involucrara a los mismos sería inútil y pecaminoso (Ceccarelli 2006).

Esta tradición -que Leibniz conocía, como ya se dijo, al menos en la obra de Summenhart- tendrá consecuencias en la manera en la que el mundo protestante comprenderá el asunto moral de los seguros y las apuestas en general. Si bien los juegos de azar proliferaban en el siglo XVII en Inglaterra, la burguesía protestante los condenaba con mayor fuerza que la tradición católica. Los argumentos, sin embargo, eran muy semejantes a los propuestos por la escolástica en la línea de Summenhart: lucrar con los objetos de la providencia divina no sólo es pecaminoso por vender algo que no se posee (un hecho futuro), sino porque implica algo que es muy contrario al espíritu protestante, a saber, el deseo de conseguir riquezas por un medio distinto al del trabajo dedicado, esforzado y mundano (Reith 2006, 134).

Estas ideas estarán ampliamente difundidas entre la comunidad protestante tanto en Inglaterra como en Alemania. La resistencia a aceptar los juegos de azar llevaba a la inevitable conclusión de que, por las mismas razones, un contrato de seguros no podía ser aceptable. ${ }^{16}$ Como ya se mencionó, el conde de Oldenburg se había negado a establecer un sistema de seguros precisamente por representar este acto un desafío a la providencia divina, y las empresas privadas de Inglaterra recibían constantemente acusaciones semejantes (Thomann y von der Schulenburg 2010, 47). ${ }^{17}$

\section{LA SOLUCIÓN DE LEIBNIZ}

Ciertamente, la relación entre juegos de azar y seguros podría no parecer muy evidente a un lector contemporáneo, pues la esencia entre este tipo de contratos parece ser opuesta. Si la naturaleza de los seguros consiste en eliminar el riesgo de una transacción o un proceso, los juegos de azar parecen buscar el riesgo como un fin. Sin embargo, nuestra perspectiva actual de los seguros es muy diferente a lo que implicaba un contrato de este tipo. Antes del siglo XVIII no existían las herramientas matemáticas ni de recolección de datos para generar los cálculos sin los cuales sería imposible imaginar la industria de seguros en nuestros días. Antes de la aparición de estas herramientas y de su uso activo en

16 Vale la pena recordar que ni los juegos de azar ni los seguros dejaron de existir, pero el discurso moral en torno a ellos fue cambiando muy lentamente, principalmente en el mundo protestante, en donde no resonó con fuerza la idea de la apuesta como un contrato no fraudulento entre iguales.

17 Objeciones semejantes se lanzaban contra un modelo de "protoseguro" denominado "fondos de viudas" que fueron muy populares en Alemania y, particularmente, en Hannover, durante el siglo XVIII (Rosenhaft 2010, 28). 
el cálculo de los seguros, los contratos de aseguramiento se hacían en un contexto fuertemente azaroso. En palabras de Rosenhaft, hay en la historia de los seguros un paso que va del risk-taking al risk-avoidance (Rosenhaft 2010, 1718).

Pero este cambio no era posible fácticamente sino hasta que se lograra hacer un cálculo verosímil de este riesgo. No será sino hasta bien entrado el siglo XVIII que se podrá comprender la incertidumbre como pérdida y, en ese sentido, a la neutralización del riesgo como un valor en sí mismo que aportaría el asegurador (Neary y Taylor 2006, 345-47).

De alguna manera, Leibniz es un pionero en los esfuerzos para llevar el ámbito del riesgo al ámbito de la certeza. Son bien conocidos los aportes en el ámbito de la probabilidad y la estadística por parte del filósofo de Hannover. Si bien en el tema particular de los seguros Leibniz no se muestra muy entusiasta en la aplicación de los descubrimientos recientes sobre la estadística, sí que ve en ellos una vía clara y adecuada para mejorar la vida de las personas y de la sociedad. Durante los años de la elaboración de los textos sobre los seguros, Leibniz se encuentra en una intensa polémica con Johann Bernoulli sobre la viabilidad de los descubrimientos probabilísticos del autor suizo, y se observa en el autor alemán una cierta resistencia a dar uso a estas herramientas matemáticas (Thomann y von der Schulenburg 2010, 45). Sin embargo, Leibniz sí realizará esfuerzos por expresar matemáticamente detalles de probabilidad en juegos, esperanza de vida y, principalmente, rentas vitalicias. En general, no se pueden leer sus dudas sobre los métodos probabilísticos de Bernoulli como una negativa a emplear por principio las matemáticas para asuntos tan complejos y aleatorios como los de los riesgos imprevistos. Todo lo contrario. El siguiente texto es elocuente respecto a esta convicción:

Niegan algunos que el Derecho sea una ciencia porque, dicen, no trata de cosas de carácter necesario sino de cosas que suceden de ordinario, siendo así que la ciencia lo es de cosas eternas. Yo creo que las razones del Derecho son también eternas y que se da ciencia de la verdad eterna en las cosas que suceden de ordinario si se conoce la razón de las mismas y el grado de probabilidad, como nos han enseñado los matemáticos con sus demostraciones sobre la suerte (An jus naturae aeternum?, Grua 638 / Andreu III, 1, 166).

En su trabajo como diplomático, Leibniz explicó en distintas ocasiones la necesidad de establecer un sistema de aseguramiento contra desastres naturales. Así se lo hizo saber al duque Johann Friederich (Knob., I.1, 4) y al emperador del Sacro Imperio Romano Germánico (Knob., I.2, 8). De la misma manera, 
cuando visitó en 1711 al Zar de Rusia, Pedro el Grande, le informó de los posibles beneficios de un sistema así (Rudolph 2018, 559).

Quisiera mostrar a continuación cómo la discusión sobre la moralidad de los seguros, en la que la Escuela de Salamanca tiene un papel preponderante, está presente en la solución que propone Leibniz para la administración de este tipo de contratos. Para ello, se abordarán las dos vertientes del problema que habían sido ya mencionadas. Dado que Leibniz no aborda concretamente, como se dijo al comienzo, el problema de los seguros, nos serviremos de otras dos figuras financieras relevantes para comprender mejor las ideas que están detrás de los contratos de aseguramiento: las rentas vitalicias y el Monte de Piedad.

\section{EL PROBLEMA DE LA USURA}

A diferencia de los autores que hemos mencionado, Leibniz no considera los contratos de seguros como contratos entre particulares, sino como una institución que debería estar gestionada por el estado. ${ }^{18} \mathrm{El}$ sistema de seguros no sólo debería ser público, sino que - propone Leibniz- convendría que estuviera coordinado por las sociedades científicas que él mismo había impulsado de distintas maneras (Knob. I.5, 25).

Leibniz tiene una versión mucho más acotada de la usura que la de los autores salmantinos. Sin dejar de censurar la usura, Leibniz comprende que el cobro de intereses no implica necesariamente caer en este pecado:

El interusurio, o descuento en la amortización anticipada, en alemán vulgar Rabat es la diferencia entre una suma debida en un cierto día y su valor presente, es decir, cuánto más exigiría a medida que pasa el tiempo, o bien cuál sería su disminución equitativa, si se reembolsa ahora lo que se debería reembolsar dentro de varios años (Knob. $272^{19} /$ OFC 7B, 842). ${ }^{20}$

18 Recordemos que, como se mencionó arriba, Leibniz había tenido ya contacto directo con aseguradoras privadas en Inglaterra.

19 Meditatio jurídico-mathematica de interusurio simplice

20 Domingo de Soto, siguiendo a Aquino (S. Th. II-II, q. 78, a. 2), admitía lo que hoy se conoce como intereses moratorios, que serían un pago al daño ocasionado por el retraso en la devolución del préstamo (Soto, VI, q. 1, a. 3). El concepto de daño es suficientemente ambiguo en los textos del salmantino, por lo que se podrían argumentar como morales algunos cobros de intereses simples. Sin embargo, líneas después, se censura completamente considerar a estos daños como la incapacidad de hacer rendir el dinero prestado mientras está lejos de las manos del prestamista. Domingo de Soto ya consideraba los casos en los que los bienes prestados, ya sea de especie o de dinero, cambian su valor en el tiempo. La solución está relacionada con la distinción entre venta y préstamo (cfr. Soto, VI, q. 1, a. 2). 
Es evidente que la idea de que el dinero es en sí mismo estéril va siendo dejada de lado. El dinero adquiere o pierde valor en el tiempo. Probablemente la discusión en la que esta convicción se hace más evidente es en el tratamiento de las rentas vitalicias (cfr. AA IV, 3, 439-46 ${ }^{21}$ / OFC 7B, 831-40; Knob., III.11, 446-8722 / OFC 7B, 865-91; Knob., III.2, 324-723 / OFC, 7B, 879-83). Leibniz elabora grandes proyectos financieros del pago de rentas vitalicias por parte de los Estados para lograr un rápido financiamiento del gobierno. Las rentas vitalicias eran acuerdos financieros en los que una persona determinada sometía sus bienes (inmuebles o capital) a una renta que duraría mientras esta persona viviera. Se trata, sobre todo, de bienes que, a efectos prácticos, aportarían una renta eterna. Las rentas vitalicias permitirían intercambiar esta renta eterna y, en su lugar, recibir una renta mucho mayor en cantidad, pero durante un tiempo menor, a saber: lo que le quedara de vida al propietario; esto les permitiría garantizar mayores ingresos a lo largo de su vida, ya sea porque las rentas fijas de sus bienes fueran insuficientes, ya porque no tuvieran a quién heredar dichos bienes. Lo que sigue a la propuesta de Leibniz sobre las rentas vitalicias es un interesante argumento matemático para calcular cómo estimar la reducción de rentas presuntamente eternas a una distribución finita en una serie de años. Bajo este esquema se vería beneficiado el Estado pues, aunque pagaría rentas altas en un principio, éstas se extinguirían pronto; y se verían beneficiados también los particulares, pues gozarían en vida la renta de aquello que habría de redituar después de su muerte. Leibniz divide así a los actores económicos en personas inmortales y personas mortales: los primeros son aquellos cuyos bienes, y su voluntad sobre ellos, sobrepasa su muerte; los segundos son aquellos cuyo domino sobre dichos bienes muere con ellos. De esta manera, piensa Leibniz, "todo esto da lugar a un comercio asombroso, realmente admirable y de gran utilidad pública y privada, a saber, un comercio entre personas mortales e inmortales" (AA IV, 3, 442 24 / OFC, 7B, 834). Las personas inmortales se asemejan a lo que llamaríamos hoy personas morales: son estados, instituciones, iglesias, ciudades, etc. ${ }^{25}$

El tratamiento de las rentas vitalicias deja ver tres convicciones claras de Leibniz: a) que el dinero en el tiempo tiene un valor intrínseco y que, en ese sentido, el cobro de intereses no es necesariamente usura; b) que ese valor del tiempo puede ser correctamente estimado mediante recursos matemáticos; y c)

21 De reditibus ad vitam...

22 De aestimatione de reditum ad vitam

23 Loss und Leibtrenten

24 De reditibus ad vitam...

25 Aunque una persona cuyos bienes puedan ser heredados y redituar eternamente se convertiría también en una persona eterna. 
que los beneficiarios de ese acuerdo son principalmente los particulares y el Estado.

La vinculación entre las rentas vitalicias y los seguros es evidente: en ambos casos se trata de un contrato con un alto grado de aleatoriedad. En el caso de las rentas vitalicias, Leibniz propone un cálculo probabilístico (bastante primitivo, ciertamente) de cómo se puede estimar la esperanza de vida de las personas. ${ }^{26}$ Es una apuesta en la que, dado el cálculo probabilístico, si el individuo muere antes de la fecha estimada, el Estado gana; de lo contrario, es el individuo el que resulta beneficiado.

Ahora bien, si se acepta esta extrapolación entre los seguros y las rentas vitalicias, bajo un esquema moral restrictivo (piénsese, por ejemplo, en Summenhart), ambas instituciones serían censuradas tanto por usura como por implicar al azar en intercambios financieros. ¿Cómo un planteamiento de este tipo se podría salvar de estas acusaciones que, como se ha mostrado, Leibniz tenía presentes?

A mi juicio, el carácter público de las instituciones financieras es central en la propuesta de Leibniz. Se observa tanto en lo dicho sobre las rentas vitalicias como en el tratamiento de los seguros como en otra discusión también importante: el Monte de Piedad. En De l'Etablissement des Monts de Piété (Knob., IV.1, 536-42), ${ }^{27}$ Leibniz argumenta que el cobro de intereses en los Montes de Piedad "no es usura pues no hay en él ganancia sin resolución. Puesto que la usura es una ganancia que proviene del préstamo sin resolución y con pacto; quien es indemnizado por cesación de ganancias, o aparición de perjuicios, salario por sus esfuerzos y compensación de gastos no es usurero".

Me parece que un tema central en las tres instituciones mencionadas es la publicidad del financiamiento y del retorno de intereses. Dado que Leibniz se encuentra ya en un ambiente en donde la línea entre el uso del dinero con fines lucrativos y su uso en las necesidades básicas es cada vez más borrosa, su solución no es establecer una casuística que indique en qué casos se comete usura y en qué casos no, en qué casos hay cesación de ganancias y en qué casos no. ${ }^{28}$

26 Algunos textos sobre el tratamiento estadístico de la esperanza de vida de las personas son De longevitate, Knob., III.12, 488-95; Essay de quelques raisonnementes..., Knob., III.10, 428-45.

27 Entre los salmantinos y conimbricenses que cita Leibniz a su favor en el texto, se encuentran Luis de Molina, Fernando Rebello, Francisco Sarmiento, Juan Azor, además de otros escolásticos como el Cardenal Cayetano, V. Regnauld, Vicenzo Filliucci, T. Buoninsegni. Leibniz no cita a Soto, pero bien podría haberlo enumerado entre los detractores.

28 En este sentido, es prototípica la división entre usura mental y usura real que proponían algunos moralistas. Como ejemplo, cfr. de Santa Teresa, Marcos, Compendio moral salmaticense según la mente del Angélico Doctor, XX, 3, 2. Se trata de un compendio del manual elaborado por Antonio de San Joseph en el siglo XVIII. 
Prefiere desplazar esta sospechosa ganancia al Estado para que la situación de la gente vulnerable no implique, de manera estructural, una ganancia para otros particulares con más fortuna. Leibniz no hace una demostración de esto, pero un pasaje sobre los Montes de Piedad, que desborda pragmatismo, es muy elocuente:

OBJECIÓN 4 [formulada por algunos contra los Montes de Piedad]: Se dice que los montes impedirán el comercio porque las personas encontrarían ahí un medio mejor de emplear su dinero. Pero es como si se dijese que la lluvia secaría la nube. Los particulares que puedan encontrar dinero y no estén arruinados por la usura estarán mejor situados para ganarse la vida, la pobreza impide a la gente hacer bien las cosas.

Las usuras son desorbitadas, pero ¿cuál es el medio de exterminarlas sin aportar un remedio? Es como decirle a un enfermo: esté usted bien. La constitución de las rentas sólo se consigue tras largos periodos de tiempo y la obligación de no sacar provecho de ellas las hace difíciles (Knob., IV.1, 542 ${ }^{29}$ / OFC 7B, 896-7).

Este tono tan consecuencialista sólo es comprensible si se atiende a la idea de "bien común", sobre la cual merece la pena ofrecer unas breves líneas.

\section{EL BIEN COMÚN}

Leibniz propone trasladar la presunta "usura" del beneficio particular al del estado. Se consigue así, precisamente, reducir la usura entre particulares y elevar el bien común de los ciudadanos. La justificación de ello es que no hay bien público sin bien privado ni viceversa:

Así pues, los que consideran el problema desde el punto de vista de la verdad, entienden que la ciencia de lo justo y la de lo útil, esto es, del bien público y del privado, están mutuamente implícitas, y que difícilmente puede uno ser feliz en medio de una masa de desgraciados (AA VI, 1, 460 / Guillén, 69).

En efecto, la idea de bien común en Leibniz es fundamental para su visión de la política, del derecho y de la moral. Este concepto ha sido ya ampliamente retratado en la literatura secundaria. Me interesa rescatar aquí dos aspectos del mismo: a) que el paso de la caridad a la justicia implica sabiduría; b) que el

29 De l'Etablissement des Monts de Piété

30 Elementa juris naturalis 
iusnaturalismo leibniziano implica un cierto consecuencialismo y un cierto comunitarismo.

El primer asunto se ve claramente reflejado en el siguiente texto:

LA CARIDAD es una benevolencia general. Y LA JUSTICIA es la caridad conforme a la sabiduría. Así, cuando uno tiene ganas de querer hacer cuanto dependa de uno para que sea feliz todo el mundo, se tiene caridad. Y cuando la misma está bien regulada por la sabiduría, de suerte que nadie pueda quejarse, resulta de ahí la virtud llamada justicia, con objeto de que no se haga daño sin necesidad a nadie y se haga antes bien, en cuanto pueda uno; mas, sobre todo, allá donde esté mejor empleado, y perfecto y más amable. (Grua, $\left.125^{31}\right)$.

Me interesa resaltar que no basta la caridad, sino que es necesaria la sabiduría para regularla de manera adecuada. Así, se reafirma que la justicia depende de una cierta medida o regulación y, en consecuencia, el bien común sólo es asequible mediante un cálculo cada vez más refinado. La instrumentación estadística no puede, en consecuencia, dejarse completamente de lado. En el siguiente apartado se verá en qué manera es que Leibniz inserta concretamente este cálculo probabilístico.

En segundo lugar, resulta interesante que Leibniz admita un cierto consecuencialismo comunitarista en el corazón de su iusnaturalismo: las acciones humanas son evaluadas moralmente por las consecuencias que tienen en su comunidad. ${ }^{32}$ Bajo ciertos límites - pues Leibniz no considera que el bien común deba merecer sacrificios excesivos o heroicos-el bien común debe estar por encima del bien particular. ${ }^{33}$ No se trata de que el primero aniquile al segundo, sino que haya una moderación prudente de la mayoría de los ciudadanos que permita la maximización del bien. ${ }^{34}$

Una aseguradora pública cumpliría cabalmente con estos requisitos. Es precisamente un instrumento que permite distribuir uniformemente un riesgo entre

$31 \quad$ La felicité

32 Esta tesis es defendida en Txetxu Ausín y Lorenzo Peña, "Derecho y Bien Común En Leibniz (Una Apología de La Fraternidad)," en Ciencia, Tecnología y Bien Común: La Actualidad de Leibniz, eds. A. Andreu (et. al) (Valencia: Universidad Politécnica de Valencia, 2002), 321.

33 Como afirma Roberto Casales, para Leibniz, en última instancia, el único bien real es el bien común. Roberto Casales García, Justicia, amor e identidad en la ontología monadológica de Leibniz (Granada: Editorial Comares, 2018), 123.

34 Cfr. Ausín and Peña, 19; Casales García, Justicia, amor e identidad en la ontología monadológica de Leibniz, 124. Leibniz afirma sobre la justicia que "es la prudencia con la que no perjudicamos con ningún daño a los demás, y con lo que nos convertimos en causa de un beneficio" Elementa juris naturalis, A VI, 1; Grua 640 / Guillén, 13. 
toda la población, de manera que la mala fortuna no sea desproporcionada para algunos pocos, aunque la mayoría de los ciudadanos se vean privados de algunos pocos bienes para sufragar esas tragedias. Así, una institución aseguradora pública cumpliría con los criterios requeridos por Leibniz en torno al bien común: evalúa moralmente las acciones particulares conforme a las consecuencias que puede tener en la comunidad (b), ${ }^{35} \mathrm{y}$ esta evaluación la realiza mediante un cálculo estadístico que garantiza la justicia distributiva (a).

\section{EL PROBLEMA DE LA PROVIDENCIA}

Por otra parte, Leibniz también da respuesta al problema de la providencia. Hacer una descripción de este concepto sería excesivo para los intereses del presente artículo. Baste con mencionar que Leibniz considera que la providencia se da desde fuera del tiempo: Dios creó este mundo con todas sus propiedades determinadas, por lo que cada uno de los estados (incluso los futuros) están determinados ex hypothesi. ${ }^{36}$ Por esta razón, Leibniz se mostrará menos inclinado a poner reparos a los esfuerzos humanos de anticipar dicha providencia, pues, por decirlo de algún modo, los "dados ya están echados" de cualquier manera.

Dejando de lado las particularidades de la interpretación leibniziana de la providencia, volvamos al tema de las aseguradoras. Se había mostrado cómo los seguros, lo mismo que otros contratos donde se involucraba el azar, se consideraban pecaminosos por apropiarse de la providencia y voluntad divinas, esto es, de eventos futuros cuya factibilidad sólo Dios conocía. Esta complicación existe ciertamente en un contrato entre dos particulares (entre dos personas mortales): no se conoce el resultado final de los dados o la cantidad de años que vivirá un sujeto en un contrato de renta vitalicia o si la casa asegurada se incendiará o si el seguro se pagará en balde. Sin embargo, cuando se incluyen personas inmortales (el Estado, por ejemplo) el riesgo y la incertidumbre se minimiza. La posibilidad de incluir personas inmortales en la ecuación permite que el riesgo (incognoscible) tienda a una constante (cognoscible) al multiplicar los casos; de la misma manera en que el resultado de cinco monedas lanzadas al aire es incierto, pero se sabe que, tras 1000 lanzamientos, se tendrá un resultado cercano al de $50 \%$ de las caras y $50 \%$ de las cruces. Las personas inmortales pueden entonces, sin necesidad de conocer la providencia divina (esto es, sin saber si esta persona

35 Recordemos que, si bien Domingo de Soto tenía argumentos consecuencialistas semejantes para el caso de los seguros, en términos generales los autores escolásticos evaluaban la acción en sí misma bajo los criterios ya vistos y no por sus consecuencias.

36 Cfr. Discours de métaphysique, §XIII, A VI, 4, 1547 (OFC II, 173); De contingentia, A VI, $4,1649-1652$. Esto no implica necesariamente un determinismo, pues los hechos de este mundo no son necesarios en la medida en que su contrario no implica contradicción. 
ha de morir o aquella casa se ha de incendiar), tener elementos para evaluar y valuar económicamente el riesgo. Recordemos el texto leibniziano referido antes: "Yo creo que las razones del Derecho son también eternas y que se da ciencia de la verdad eterna en las cosas que suceden de ordinario si se conoce la razón de las mismas y el grado de probabilidad, como nos han enseñado los matemáticos con sus demostraciones sobre la suerte" (Grua, $638^{37}$ ).

Recordemos que Domingo de Soto, en continuidad con el contractualismo de Olivi, dejaba a los particulares definir el precio a pagar por los seguros: "nosotros no discutimos por el precio; puede, efectivamente ser justo, o injusto. Esto que lo vean los contratantes" (Soto, VI, q. 7). Soto era consciente de que no existían las herramientas para establecer el precio natural de un servicio de esta índole: ¿cómo calcular los riesgos de esta nave, que partirá este día, a través de estos mares concretos? Leibniz, con la ciencia de la probabilidad apenas naciendo, tampoco poseía las herramientas para responder a algo así. Sin embargo, concentrando todos los contratos en una entidad y preservando esta entidad a lo largo del tiempo se tiene un doble rédito: en primer lugar, que no hay incentivo para generar usura en el contrato y que, incluso dado el caso, esa "posible" usura involuntaria sería redituada al bien común; en segundo lugar, que no hace falta conocer la providencia divina de la suerte de cada particular, sino las leyes generales que guían esa suerte y "la razón" de las mismas. El primero de estos réditos responde al problema de la usura; el segundo responde al problema de la providencia. Ambas apuntan al bien común como el elemento de cohesión y razón última de los contratos e intercambios entre particulares; como reza el título de este trabajo, "el bien público debe jugar siempre el papel más importante" (De reditibus ad vitam ..., Knob., III.6, 384-5).

\section{CONCLUSIÓN}

Se ha mostrado que la moralidad y la legalidad de los contratos de seguros gozó de una nutrida discusión desde los comienzos de la tradición escolástica, pasando por la Escuela de Salamanca, hasta bien entrado el siglo XVIII.

Leibniz se inserta en esta discusión de manera implícita. Si bien no hace referencia explícita a esta tradición (principalmente porque sus textos sobre los seguros son de corte diplomático y no académico), hay evidencia textual, como se mostró en la segunda sección de este trabajo y a lo largo del artículo, de que estaba bien consciente de los detalles de esta discusión. Además, Leibniz

37 An jus naturae aeternum? 
enmarca todos sus proyectos bajo los ideales del irenismo y la reunificación de las Iglesias. En este sentido, estaba obligado a dar respuesta tanto a la tradición católica como a la tradición protestante.

Como se mostró, hay dos grandes problemas que los moralistas salmantinos y escolásticos en general encontraron en torno a los seguros: el problema de la usura y el problema de la providencia divina. La respuesta de Leibniz consiste en proponer que las instituciones financieras que lucraban con el riesgo sean públicas: de esa manera no hacía falta asumir el riesgo en tanto incognoscible, ni se lucraba con la providencia divina, y, por otra parte, la presunta "usura" se ve diluida o retribuida en favor del bien común, que es condición de posibilidad para la felicidad y la vida pública.

\section{REFERENCIAS BIBLIOGRÁFICAS}

Aquino, Tomás de. Suma Teológica. Editado por Francisco Barbado. Biblioteca de Autores Cristianos, 2010.

Aristóteles. Política. Madrid: Gredos, 1988.

Ausín, Txetxu y Lorenzo Peña. "Derecho y bien común en Leibniz (una apología de la fraternidad)". En Ciencia, tecnología y bien común: la actualidad de Leibniz. Editado por A. Andreu (et. al), 320-39. Valencia: Universidad Politécnica de Valencia, 2002.

Casales García, Roberto. Justicia, amor e identidad en la ontología monadológica de Leibniz. Granada: Editorial Comares, 2018.

Ceccarelli, Giovanni. "Risky Business: Theological and Canonical Thought on Insurance from the Thirteenth to the Seventeenth Century". Journal of Medieval and Early Modern Studies 31/3 (2001): 607-58.

-. "Gambling and Economic Thought in the Late Middle Ages". Ludica, Annali Di Storia e Civiltà Del Gioco 12 (2006): 54-63.

Cruz Barney, Oscar. El riesgo en el comercio hispano-indiano: préstamos y seguros marítimos durante los siglos XVI a XIX. México: Universidad Nacional Autónoma de México, 1998.

Garber, Daniel. Leibniz: Body, Substance, Monad. New York: Oxford University Press, 2011.

Grabill, Stephen J. Sourcebook in Late-Scholastic Monetary Theory: The Contributions of Martin de Azpilcueta, Luis de Molina, S.J., and Juan de Mariana, S.J. Lanham: Lexington Books, 2007.

Gregorio IX. "Naviganti”. Decretalium Compilatio. 2007. http://www.intratext.com/IXT/LAT0833/_P1BL.HTM. 
Knobloch, Eberhard. "Leibniz's Mathematical Handling of Death, Catastrophes, and Insurances". Matematica, cultura e società 1/3(2016): 259-74.

-. "Leibniz's Contributions to Financial and Insurance Mathematics". En International Conference on Analytical and Computational Methods in Probability Theory and Its Applications, 2017.

Leibniz, G. W. Die Werke von Leibniz. Editado por O. Klopp. Hannover, 1864.

-. Sämtliche Schriften und Briefe. Berlin: Akademie Verlag, 1923.

- G. W. Leibniz: Textes inédits d'après les manuscrits de la Bibliothèque Provinciale de Hanovre. Editado por G. Grua. Paris: PUF, 1948.

- Die philosophischen Schriften. Editado por C. J. Gerhardt. Hildesheim: Olms, 1965.

—. Escritos filosóficos. Editado por Ezequiel de Olaso. Buenos Aires: Editorial Charcas, 1982.

- Los elementos del derecho natural. Editado por Tomás Guillén. Madrid: Tecnos, 1991.

-. Hauptschriften zur Versicherungs- und Finanzmathematik. Editado por Eberhard Knobloch. Berlin: Akademie Verlag, 2000.

-. Methodus vitae (escritos de Leibniz). Editado por Agustín Andreu. Valencia: Editorial UPV, 2001.

- G. W. Leibniz. Obras filosóficas y científicas. Editado por Juan Nicolás. Granada: Comares, 2007.

Mercer, Christia. "Mechanizing Aristotle: Leibniz and Reformed Philosophy". En Studies in Seventeenth-Century Philosophy, editado por M.A. Stewart, 117-52. Oxford: Clarendon, 1997.

Mora, Marisol De. "Pensiones, rentas y seguros. Los primeros cálculos y la participación de Leibniz". En Historia de la probabilidad y de la estadística, 35-48. Madrid: Editorial ACE, 2002.

Neary, Mike; Taylor, Graham. "From the Law of Insurance to the Law of Lottery". En The Sociology of Risk and Gambling Reader. Editado por James F. Cosgrave, 339-54. New York, London: Routledge, 2006.

Pearson, Robin. "Fire, Property Insurance, and Perceptions of Risk in Eighteenth-Century Britain". En The Appeal of Insurance. Editado por Geoffrey W. Clark y Gregory Anderson. Toronto: University of Toronto Press, 2010.

Pelletier, Arnaud. "Leibniz and the Scholastics". Studia Leibnitiana 46/2 (2014): 123-26.

Reith, Gerda. "The Pursuit of Chance". En The Sociology of Risk and Gambling Reader. Editado por James Cosgrave, 125-42. New York, London: Routledge, 2006. 
Rosenhaft, Eve. "Taming Chance: Risk and the Quantification of Uncertainty". En The Appeal of Insurance. Editado por G. W. Clark y G. Anderson, 16-

42. Toronto: University of Toronto Press, 2010.

Rossi, Guido. Insurance in Elizabethan England: The London Code. Cambridge: Cambridge University Press, 2016.

Rudolph, Hartmut. "Scientific Organization and Learned Societies". En The Oxford Handbook of Leibniz. Editado por Maria Rosa Antognazza, 543-61. New York: Oxford University Press, 2018.

Soto, Domingo de. Tratado de la justicia y el derecho. Madrid: Instituto de Estudios Políticos, 1968.

Summenhart, Conrad. De contractibus licitis atque illicitis tractatus. Venecia: Franciscum Zilettum, 1580.

Thomann, Christian; von der Schulenburg, J. Matthias Graf. "Gottfried Wilhelm Leibniz's Work on Insurance". En The Appeal of Insurance, editado por G. W. Clark y G. Anderson, 43-51. Toronto: University of Toronto Press, 2010.

Leonardo Ruiz-Gómez Facultad de Filosofía Universidad Panamericana Augusto Rodin 498, Insurgentes Mixcoac 03920 Ciudad de México (México) https://orcid.org/0000-0002-8229-713X 